Bundesgesundheitsbl 2013 · 56:668-677

DOI 10.1007/s00103-012-1662-5

Online publiziert: 27. Mai 2013

๑) Springer-Verlag Berlin Heidelberg 2013

\section{Additional material online}

An English full-text version of this article is available at SpringerLink under supplementary material: dx.doi.org/10.1007/s00103-012-1662-5
C. Heidemann ${ }^{1}$.Y. Du ${ }^{1}$ I. Schubert ${ }^{2}$. W. Rathmann ${ }^{3}$. C. Scheidt-Nave ${ }^{1}$

${ }^{1}$ Abteilung für Epidemiologie und Gesundheitsmonitoring, Robert Koch-Institut, Berlin

2 PMV Forschungsgruppe an der Klinik und Poliklinik für Psychiatrie und Psychotherapie des Kindes- und Jugendalters, Universität zu Köln

${ }^{3}$ Deutsches Diabetes-Zentrum, Institut für Biometrie und Epidemiologie, Leibniz-Zentrum für Diabetes-Forschung an der Heinrich-Heine-Universität, Düsseldorf

\title{
Prävalenz und zeitliche Entwicklung des bekannten Diabetes mellitus
}

\section{Ergebnisse der Studie zur Gesundheit Erwachsener in Deutschland (DEGS1)}

\section{Hintergrund und Fragestellung}

Diabetes mellitus ist eine chronische Stoffwechselerkrankung, die durch erhöhte Blutzuckerkonzentrationen infolge eines absoluten oder relativen Insulinmangels gekennzeichnet ist. Ein absoluter Insulinmangel charakterisiert den „Typ-1-Diabetes“, der sich überwiegend im Kindes- und Jugendalter manifestiert. Zugrunde liegt eine autoimmune Zerstörung der Insulin-produzierenden Betazellen der Bauchspeicheldrüse [1]. Ein relativer Insulinmangel kennzeichnet den „Typ-2-Diabetes“, der vorwiegend im Erwachsenenalter auftritt.

Der gestörte Glukosestoffwechsel resultiert dabei aus einer verminderten Insulinwirkung in Verbindung mit einer unzureichend kompensierenden Insulinsekretion. Neben einer genetischen Prädisposition zählen eine durch den „westlichen“ Lebensstil geprägte Ernährungsweise, Bewegungsmangel und damit im Zusammenhang stehendes Übergewicht $\mathrm{zu}$ den wesentlichen Risikofaktoren [1, 2]. Ein relativer Insulinmangel kann erstmals in der Schwangerschaft auftreten und bildet sich danach in der Regel wieder zurück („Schwangerschaftsdiabetes"). Es verbleibt jedoch ein hohes Risiko, im weiteren Verlauf des Lebens einen manifesten Typ-2-Diabetes zu entwickeln [1].

Lang anhaltend erhöhte Blutzuckerkonzentrationen bei einem noch nicht diagnostizierten Diabetes oder einem unzureichend kontrollierten diagnostizierten Diabetes führen zu Schädigungen der Blutgefäße und peripheren Nerven. Diese resultieren in einem erhöhten Risiko für kardiovaskuläre Erkrankungen wie Herzinfarkt und Schlaganfall sowie für Nierenversagen, Erblindung und Fußamputationen $[1,3]$. Diese Folgeerkrankungen führen neben der Diabeteserkrankung selbst zu einer verminderten Lebensqualität und reduzierten Lebenserwartung bei den Betroffenen $[3,4]$ sowie zu hohen Kosten für das Gesundheitssystem [5].

Internationale Analysen zeigen, dass sich die Zahl der Erwachsenen mit Diabetes in den letzten 3 Jahrzehnten weltweit mehr als verdoppelt hat [6]. Überwiegend reflektiert diese Entwicklung die Zunahme des Typ-2-Diabetes [2]. Bei etwa der Hälfte der Betroffenen ist die Erkrankung vermutlich bislang nicht diagnostiziert [7].

In Deutschland durchgeführte Studien zur Einschätzung der Prävalenz des bekannten Diabetes basieren meist auf Daten von regionalen Studien, Kranken- versicherungen oder Hausarztpraxen, die 5 bis 10 Jahre oder länger zurückliegen (s. Übersicht in $\bullet$ Tab. 6). Bundesweit bevölkerungsbezogene Daten zum bekannten Diabetes lieferten zuletzt die telefonischen Surveys „Gesundheit in Deutschland aktuell“ (GEDA) 2009 [8, 9] und GEDA 2010 [10]. Studien zum nicht diagnostizierten Diabetes liegen nur vereinzelt vor [11].

Auf Datengrundlage des Befragungsund Untersuchungssurveys von 20082011 werden im Folgenden aktuelle, repräsentative Einschätzungen zur Lebenszeitprävalenz des bekannten Diabetes in der 18- bis 79-jährigen Wohnbevölkerung Deutschlands dargestellt. Im Vergleich zum letzten Befragungs- und Untersuchungssurvey von 1997-1999 wird zudem deren zeitliche Entwicklung abgebildet. Die Ergebnisse werden im Kontext bisheriger Studien in Deutschland dargestellt und diskutiert. Zur Einschätzung der Prävalenz und zeitlichen Entwicklung des nicht diagnostizierten Diabetes sind über die Zeit vergleichbare Labordaten erforderlich, die derzeit für beide Surveys kreuzkalibriert werden und daher noch nicht Bestandteil des vorliegenden Beitrages sind. 


\section{Methoden}

\section{Studiendesign}

Die „Studie zur Gesundheit Erwachsener in Deutschland" (DEGS) ist Bestandteil des Gesundheitsmonitorings des Robert Koch-Instituts (RKI). Konzept und Design von DEGS sind an anderer Stelle ausführlich beschrieben $[12,13,14,15,16]$. Die erste Erhebungswelle (DEGS1) wurde von 2008 bis 2011 durchgeführt und umfasste Befragungen, Untersuchungen und Tests [17, 18]. Zielpopulation war die in Deutschland lebende Bevölkerung im Alter von 18 bis 79 Jahren. DEGS1 hat ein Mischdesign, das gleichzeitig querund längsschnittliche Analysen ermöglicht. Hierbei wurde eine Einwohnermeldeamtsstichprobe durch ehemalige Teilnehmerinnen und Teilnehmer des „Bundes-Gesundheitssurveys 1998“ (BGS98) ergänzt. Insgesamt nahmen 8152 Personen teil, darunter 4193 Ersteingeladene (Response 42\%) und 3959 ehemalige Teilnehmerinnen und Teilnehmer des BGS98 (Response 62\%). 7238 Personen besuchten eines der 180 Untersuchungszentren, 914 wurden ausschließlich befragt. Die Nettostichprobe $(n=7988$, davon 7116 in Untersuchungszentren) ermöglicht für den Altersbereich von 18 bis 79 Jahren repräsentative Querschnittanalysen und Trendaussagen im Vergleich mit dem BGS98 (n=7124) [16]. Die Daten der erneut Teilnehmenden sind für Längsschnittanalysen nutzbar.

Die vorliegenden Querschnittanalysen zum bekannten Diabetes beziehen sich auf Daten der 7116 Personen im Alter von 18 bis 79 Jahren, die den DEGS1Untersuchungsteil absolviert haben. In einer zusätzlichen Analyse wird die in dieser Stichprobe ermittelte Diabetesprävalenz mit der in der Gesamt-Nettostichprobe $(n=7988)$, die auch Daten von nur Befragten enthält, verglichen. In den zeitlichen Vergleich sind die Teilnehmenden des BGS98 $(n=7124)$ eingeschlossen. Personen mit fehlender Angabe zur Diabetesdiagnose sind in den Analysen ausgeschlossen, sofern keine aktuelle Einnahme von Antidiabetika bestand (DEGS1: $\mathrm{n}=36$; BGS98: $\mathrm{n}=25$ ).

\section{Definition des bekannten Diabetes}

Zur Erfassung der Lebenszeitprävalenz des bekannten Diabetes wurde folgende Falldefinition herangezogen:

- Bejahung der Frage „Hat ein Arzt jemals bei Ihnen eine Zuckerkrankheit oder einen Diabetes festgestellt?" in einem standardisierten, computergestützten ärztlichen Interview oder

- Dokumentation der Einnahme von Antidiabetika entsprechend einer automatisierten Erfassung von in den letzten 7 Tagen eingenommenen $\mathrm{Me}$ dikamenten.

Das ärztliche Interview beinhaltete keine direkte Frage zum Diabetes-Typ. Durch folgende zusätzlich erfasste Informationen wurde jedoch der Anteil an Typ1-Diabetes abgeschätzt:

- Alter bei Diagnose <30 Jahre und

- Insulinbehandlung unmittelbar nach Diagnose und

- Insulinbehandlung derzeit.

Weiterhin wurden Frauen mit einer Diabetesdiagnose vor dem 50 . Lebensjahr in DEGS1 gefragt, ob zum Zeitpunkt der Diagnose eine Schwangerschaft vorlag. Durch folgende Verknüpfung von Informationen wurde der Anteil an Schwangerschaftsdiabetes abgeschätzt:

- Diagnose in der Schwangerschaft und

- keine aktuelle Einnahme von Antidiabetika und

- kein Bestehen des Diabetes in den letzten 12 Monaten $(n=32)$ bzw. bei fehlender Angabe $(n=6)$ oder unklarer Angabe $(n=4)$ zum Bestehen des Diabetes in den letzten $12 \mathrm{Mo-}$ naten: $\mathrm{HbA1c}<6,5 \%$ und Glukose $<7,0 \mathrm{mmol} / \mathrm{l}$ (nüchtern) $/<11,1 \mathrm{mmol} / 1$ (nicht nüchtern).

Eine Teilnehmerin mit Angabe der Diagnosestellung in der Schwangerschaft war zum Zeitpunkt des Interviews schwanger. Aufgrund vorliegender Zusatzinformationen (Diagnosestellung vor 3 Jahren, Bestehen des Diabetes in den letzten 12 Monaten, aktuelle Behandlung mit Insulin, Behandlung unmittelbar nach Diagnose durch Tabletten) wurde jedoch in diesem Fall kein Schwangerschaftsdia- betes, sondern ein Typ-2-Diabetes angenommen.

\section{Statistische Auswertung}

Die Querschnitt- und Trendanalysen werden mit einem Gewichtungsfaktor durchgeführt, der Abweichungen der Stichprobe von der Bevölkerungsstruktur (Stand 31.12.2010) hinsichtlich Alter, Geschlecht, Region und Staatsangehörigkeit sowie Gemeindetyp und Bildung korrigiert [16]. Für den Untersuchungsteil wurde ein gesonderter Gewichtungsfaktor erstellt. Bei der Berechnung der Gewichtung für die ehemaligen Teilnehmenden des BGS98 wurde die Wiederteilnahmewahrscheinlichkeit, basierend auf einem logistischen Modell, berücksichtigt. Für die Durchführung von Trendanalysen werden die Daten des BGS98 auf den Bevölkerungsstand zum 31.12.2010 altersadjustiert. Eine Nonresponder-Analyse und der Vergleich einzelner erhobener Indikatoren mit Daten der amtlichen Statistik weisen auf eine hohe Repräsentativität der Stichprobe für die Wohnbevölkerung in Deutschland hin [16].

Um sowohl die Gewichtung als auch die Korrelation der Teilnehmenden innerhalb einer Gemeinde zu berücksichtigen, wurden die Konfidenzintervalle und p-Werte mit den Survey-Prozeduren von SAS 9.2 bestimmt. Unterschiede mit pWerten $<0,05$ werden als statistisch signifikant angesehen.

Schätzungen zur Lebenszeitprävalenz in den vorliegenden Querschnitt- und Trendanalysen sind für die 18- bis 79-jährige Bevölkerung insgesamt sowie geschlechtsstratifiziert dargestellt. Weitere Stratifizierungsvariablen in Querschnittanalysen sind Lebensalter, Sozialstatus, Wohnregion und Krankenversicherung.

Der Sozialstatus wurde anhand eines Indexes bestimmt, in den Angaben zu schulischer und beruflicher Ausbildung, beruflicher Stellung sowie Haushaltsnettoeinkommen (bedarfsgewichtet) eingehen und der eine Einteilung in niedrige, mittlere und hohe Statusgruppe ermöglicht [19].

Die Wohnregion wurde für Vergleiche mit früheren Surveys in neue Bundesländer (inklusive Berlin) und alte Bundesländer stratifiziert. Zur weiteren regio- 
nalen Differenzierung wurden die alten Bundesländer in Nord (Schleswig-Holstein, Hamburg, Niedersachsen, Bremen), Mitte (Nordrhein-Westfalen, Hessen, Rheinland-Pfalz, Saarland) und Süd (Bayern, Baden-Württemberg) unterteilt.

Die Krankenversicherung wurde in gesetzliche Krankenversicherung, private Krankenversicherung als Vollversicherung und sonstige Krankenversorgung (Beihilfe, ausländische Krankenkasse, sonstiger Anspruch, keine Krankenversicherung/Selbstzahler) kategorisiert. Die gesetzliche Krankenversicherung wurde zudem analog zu Auswertungen früherer telefonischer Surveys in Allgemeine Ortskrankenkasse (AOK), Ersatzkrankenkasse, Betriebskrankenkasse und sonstige gesetzliche Krankenversicherungen unterteilt.

Durch Gegenüberstellung nicht adjustierter und altersadjustierter logistischer Regressionsmodelle wurde der Einfluss des Alters auf die Beziehung zwischen der jeweiligen Stratifizierungsvariable und der Diabetesprävalenz geprüft.

\section{Ergebnisse}

\section{Prävalenz des bekannten Diabetes}

Insgesamt gaben 591 der 7080 einbezogenen Teilnehmenden des DEGS1-Untersuchungssurveys einen jemals ärztlich diagnostizierten Diabetes an. 0 Tab. 1 zeigt die entsprechende Lebenszeitprävalenz des bekannten Diabetes für die 18bis 79-jährige Bevölkerung von insgesamt 7,2\% (Frauen: 7,4\%; Männer: 7,0\%). Die Prävalenz nimmt im Altersgang von unter $5 \%$ bei unter 50 -Jährigen bis rund $22 \%$ bei 70 - bis 79 -Jährigen bei beiden Geschlechtern deutlich zu.

Eine Analyse der Gesamt-Nettostichprobe, die auch ausschließlich Befragte berücksichtigt, führt mit 677 der 7934 einbezogenen Befragten mit einem diagnostizierten Diabetes bzw. einer Lebenszeitprävalenz von insgesamt 7,4\% (Frauen: 7,5\%; Männer: 7,2\%) zu vergleichbaren Ergebnissen.

Die ermittelte Prävalenz des Typ-1Diabetes liegt bei rund 0,1\% $(n=8)$. Dies entspricht einem Anteil von 1,1\% an der Gesamtprävalenz des bekannten Diabetes. Die Prävalenz des Schwanger-

Bundesgesundheitsbl 2013 · 56:668-677

(c) Springer-Verlag Berlin Heidelberg 2013

\section{Heidemann · Y. Du • I. Schubert · W. Rathmann · C. Scheidt-Nave \\ Prävalenz und zeitliche Entwicklung des bekannten \\ Diabetes mellitus. Ergebnisse der Studie zur \\ Gesundheit Erwachsener in Deutschland (DEGS1)}

\section{Zusammenfassung}

Die erste Welle der „Studie zur Gesundheit Erwachsener in Deutschland" (DEGS1, 20082011) ermöglicht aktuelle, repräsentative Prävalenzschätzungen des bekannten Diabetes in der 18- bis 79-jährigen Wohnbevölkerung. Im Vergleich zum „Bundes-Gesundheitssurvey 1998" (BGS98) lässt sich die zeitliche Entwicklung darstellen. Die Definition des bekannten Diabetes beruht auf Selbstangaben zu einem jemals ärztlich festgestellten Diabetes in ärztlichen Interviews oder der Einnahme von Antidiabetika. Insgesamt wurde bei 7,2\% der Erwachsenen (7,4\% der Frauen; $7,0 \%$ der Männer) jemals ein Diabetes diagnostiziert. Die Prävalenz steigt mit zunehmendem Alter deutlich an und ist bei niedrigem Sozialstatus höher als bei hohem Sozialstatus. Die Prävalenz variiert je nach Kran- kenversicherungsart und ist für Versicherte der Allgemeinen Ortskrankenkasse (AOK) am höchsten. Im Vergleich zum BGS98 zeigt sich ein Prävalenzanstieg um 38\%, wovon etwa ein Drittel auf die demografische Alterung zurückzuführen ist. Die Ergebnisse weisen im Kontext mit anderen bundesweiten Studien auf aktuell mindestens 4,6 Mio. 18- bis 79-Jährige mit einer Diabetesdiagnose hin. Geplante Analysen zum nicht diagnostizierten Diabetes werden zur Interpretation des beobachteten Prävalenzanstiegs des bekannten Diabetes beitragen.

\section{Schlüsselwörter}

Diabetes mellitus · Deutschland .

Erwachsene · Gesundheitssurvey · Prävalenz

\section{Prevalence and temporal trend of known diabetes mellitus. Results of the German Health Interview and Examination Survey for Adults (DEGS1)}

\section{Abstract}

The first wave of the "German Health Interview and Examination Survey for Adults" (DEGS1, 2008-2011) allows for up-to-date, representative prevalence estimates of known diabetes amongst the 18- to 79-yearold resident population of Germany. Temporal trends can be shown by comparing the survey findings with those of the "German National Health Interview and Examination Survey 1998" (GNHIES98). The definition of known diabetes was based on self-reports in physician-administered interviews that asked respondents if they had ever been diagnosed with diabetes by a doctor or were on anti-diabetic medication. Overall, diabetes had been diagnosed in $7.2 \%$ of the adults $(7.4 \%$ of the women; $7.0 \%$ of the men). The prevalence increased substantially with advancing age and was higher in persons of low than of high socioeconomic status. Prevalence varied de- pending on the type of health insurance held and was highest amongst those insured with AOK health insurance funds. In comparison with GNHIES98, there was a 38\% increase in prevalence, of which approximately one third is to be attributed to demographic ageing. In the context of other nationwide studies, the results indicate a figure of at least 4.6 million 18- to 79-year-olds having been diagnosed with diabetes at some point. Planned analyses of undiagnosed diabetes will contribute to the interpretation of the observed increase in the prevalence of known diabetes. An English full-text version of this article is available at SpringerLink as supplemental.

Keywords

Diabetes mellitus · Germany · Adults · Health survey Prevalence schaftsdiabetes beträgt unter Frauen 1,2\% $(n=42)$. Dies entspricht einem Anteil von $16,3 \%$ an der Gesamtprävalenz des bekannten Diabetes bei Frauen.

- Tab. 2 reflektiert für Frauen und Männer mit niedrigem Sozialstatus eine signifikant höhere Lebenszeitprävalenz des bekannten Diabetes als für solche mit hohem Sozialstatus. Dieser Unterschied ist bei Frauen stärker ausgeprägt als bei Männern. Während auch Frauen mit mittlerem Sozialstatus eine signifikant höhere Prävalenz aufweisen als solche mit hohem Sozialstatus, existieren zwischen 
Tab. 1 Lebenszeitprävalenz (Prozent, 95\%-Konfidenzintervall) des bekannten Diabetes nach Geschlecht und Altersgruppen. Nungewichtet $=7080$

\begin{tabular}{|lllllll}
\hline $\begin{array}{l}\text { Alters- } \\
\text { gruppe }\end{array}$ & $\begin{array}{l}\mathbf{1 8} \text { bis } \\
\text { 39 Jahre }\end{array}$ & $\begin{array}{l}\mathbf{4 0} \text { bis } \\
\text { 49 Jahre }\end{array}$ & $\begin{array}{l}\mathbf{5 0} \text { bis } \\
\mathbf{5 9} \text { Jahre }\end{array}$ & $\begin{array}{l}\mathbf{6 0} \text { bis } \\
\text { 69 Jahre }\end{array}$ & $\begin{array}{l}\text { 70 bis } \\
\mathbf{7 9} \text { Jahre }\end{array}$ & Gesamt \\
\hline Geschlecht & & & & & \\
\hline Frauen & 3,7 & 4,5 & 4,0 & 10,7 & 21,8 & 7,4 \\
& $(2,5-5,5)$ & $(3,0-6,8)$ & $(2,6-6,0)$ & $(8,2-13,8)$ & $(17,6-26,7)$ & $(6,5-8,5)$ \\
\hline Männer & 0,9 & 2,0 & 7,3 & 17,0 & 22,0 & 7,0 \\
& $(0,3-2,3)$ & $(1,1-3,7)$ & $(5,3-10,1)$ & $(13,1-21,7)$ & $(17,6-27,2)$ & $(6,0-8,1)$ \\
\hline Gesamt & 2,3 & 3,2 & 5,7 & 13,8 & 21,9 & 7,2 \\
& $(1,5-3,4)$ & $(2,3-4,6)$ & $(4,4-7,2)$ & $(11,4-16,6)$ & $(18,7-25,5)$ & $(6,5-8,0)$ \\
\hline
\end{tabular}

Tab. 2 Lebenszeitprävalenz (Prozent, 95\%-Konfidenzintervall) des bekannten Diabetes nach Geschlecht und Sozialstatus. $N_{\text {ungewichtet }}=7012$

\begin{tabular}{llll}
\hline Sozialstatus & Niedrig & Mittel & Hoch \\
\hline Geschlecht & & & \\
\hline Frauen & $11,6(8,6-15,5)$ & $7,4(6,3-8,7)$ & $3,0(2,0-4,5)$ \\
\hline Männer & $10,1(7,5-13,5)$ & $6,1(5,1-7,4)$ & $6,2(4,6-8,3)$ \\
\hline Gesamt & $10,9(8,8-13,5)$ & $6,8(6,0-7,7)$ & $4,8(3,7-6,0)$ \\
\hline
\end{tabular}

Sozialstatus: Index, in den Angaben zu schulischer und beruflicher Ausbildung, beruflicher Stellung sowie Haushaltsnettoeinkommen (bedarfsgewichtet) eingehen.

Tab. 3 Lebenszeitprävalenz (Prozent, 95\%-Konfidenzintervall) des bekannten Diabetes nach Geschlecht und Wohnregion. Nungewichtet $=7080$

\begin{tabular}{llllll} 
Wohnregion & $\begin{array}{l}\text { Alte Bundes- } \\
\text { länder }\end{array}$ & Darunter: & & Neue Bundes- \\
\cline { 3 - 5 } & & Nord & Mitte & Sünder & \\
\hline Geschlecht & & & & & \\
\hline Frauen & $7,1(6,0-8,4)$ & $7,1(4,6-10,9)$ & $7,7(6,3-9,4)$ & $6,3(4,6-8,6)$ & $8,7(6,7-11,3)$ \\
\hline Männer & $6,9(5,7-8,2)$ & $6,3(4,3-9,3)$ & $7,5(5,6-10,0)$ & $6,3(4,7-8,4)$ & $7,5(5,9-9,4)$ \\
\hline Gesamt & $7,0(6,2-7,9)$ & $6,7(5,2-8,7)$ & $7,6(6,3-9,2)$ & $6,3(5,1-7,8)$ & $8,1(6,9-9,5)$ \\
\hline $\begin{array}{l}\text { Alte Bundesländer: Nord: Schleswig-Holstein, Hamburg, Niedersachsen, Bremen; Mitte: Nordrhein-Westfalen, } \\
\text { Hessen, Rheinland-Pfalz, Saarland; Süd: Bayern, Baden-Württemberg. }\end{array}$
\end{tabular}

Tab. 4 Lebenszeitprävalenz (Prozent, 95\%-Konfidenzintervall) des bekannten Diabetes nach Geschlecht und Krankenversicherung. Nungewichtet $_{\text {un }}=6938$

\begin{tabular}{|llllllll}
\hline $\begin{array}{l}\text { Kran- } \\
\text { kenver- } \\
\text { siche- } \\
\text { rung }\end{array}$ & $\begin{array}{l}\text { Gesetzli- } \\
\text { che Kran- } \\
\text { kenversi- } \\
\text { cherung }\end{array}$ & \begin{tabular}{l} 
Darunter: \\
\cline { 3 - 6 }
\end{tabular} & $\begin{array}{l}\text { Allgemeine } \\
\text { Ortskran- } \\
\text { kenkasse }\end{array}$ & $\begin{array}{l}\text { Ersatz- } \\
\text { kranken- } \\
\text { kasse }\end{array}$ & $\begin{array}{l}\text { Betriebs- } \\
\text { kranken- } \\
\text { kasse }\end{array}$ & $\begin{array}{l}\text { Andere } \\
\begin{array}{l}\text { Private } \\
\text { Kranken- } \\
\text { versiche- } \\
\text { rung }\end{array}\end{array}$ & $\begin{array}{l}\text { Sonstige } \\
\text { Kranken- } \\
\text { versor- } \\
\text { gung }\end{array}$ \\
\hline Geschlecht & & & & & & \\
\hline Frauen & 7,8 & 8,6 & 7,3 & 6,8 & 8,9 & 1,2 & 4,7 \\
& $(6,7-9,0)$ & $(6,6-11,2)$ & $(5,7-9,2)$ & $(4,7-9,9)$ & $(5,7-13,6)$ & $(0,3-3,9)$ & $(2,4-9,3)$ \\
\hline Männer & 7,2 & 9,5 & 6,7 & 5,0 & 7,0 & 5,0 & 4,7 \\
& $(6,1-8,5)$ & $(7,4-12,0)$ & $(5,0-8,8)$ & $(3,2-7,7)$ & $(4,5-10,5)$ & $(2,8-8,7)$ & $(2,6-8,4)$ \\
\hline Gesamt & 7,5 & 9,0 & 7,0 & 5,9 & 7,9 & 3,8 & 4,7 \\
& $(6,7-8,4)$ & $(7,5-10,8)$ & $(5,9-8,3)$ & $(4,4-7,8)$ & $(5,7-10,8)$ & $(2,2-6,5)$ & $(3,1-7,0)$ \\
\hline
\end{tabular}

Männern mit mittlerem und hohem Sozialstatus keine Prävalenzunterschiede. Diese Ergebnisse sind auch im altersadjustierten Modell zu beobachten.

Bei Stratifizierung der Lebenszeitprävalenz des bekannten Diabetes nach Wohnregion zeigt sich entsprechend - Tab. 3 eine tendenziell höhere Prävalenz für die neuen Bundesländer im Ver-
Modell bleiben die Tendenzen bestehen, sind jedoch noch schwächer ausgeprägt.

- Tab. 4 zeigt Unterschiede in der Lebenszeitprävalenz des bekannten Diabetes nach Art der Krankenversicherung. Gesetzlich Krankenversicherte haben mit insgesamt 7,5\% eine höhere Prävalenz als Privatversicherte mit 3,8\%. Unter gesetzlich Krankenversicherten weisen AOK-Versicherte mit 9,0\% die höchste und Versicherte der Betriebskrankenkasse mit 5,9\% die niedrigste Prävalenz auf. Dieses Muster zeigt sich auch in der geschlechtsstratifizierten Analyse, wobei der Prävalenzunterschied bei Frauen zwischen den verschiedenen gesetzlichen Krankenversicherungen, bei Männern dagegen zwischen der gesetzlichen und privaten Krankenversicherung schwächer ausgeprägt ist. Im altersadjustierten Modell ist dieses Muster ebenfalls zu beobachten, die Unterschiede fallen jedoch geringer aus.

\section{Zeitliche Entwicklung des bekannten Diabetes}

In - Tab. 5 ist die Lebenszeitprävalenz des bekannten Diabetes aus dem früheren Untersuchungssurvey BGS98 für einen zeitlichen Vergleich der aus DEGS1 gegenübergestellt. Die anhand der BGS98-Daten beobachtete Lebenszeitprävalenz (gewichtet auf die Bevölkerungsstruktur 1997) betrug insgesamt 5,2\% (Frauen: 5,7\%; Männer: 4,7\%). Daraus resultiert eine signifikante Prävalenzzunahme im zeitlichen Verlauf von absolut 2,0\% (Frauen: 1,7\%; Männer: $2,3 \%$ ) und relativ $38 \%$ (Frauen: $30 \%$; Männer: 49\%). Eine signifikante Zunahme von absolut 1,4\% (Frauen: 1,3\%; Männer: $1,6 \%$ ) und relativ $24 \%$ (Frauen: $21 \%$; Männer: 30\%) besteht auch nach Berücksichtigung der demografischen Alterung der Bevölkerung (gewichtet auf die Bevölkerungsstruktur 2010). Somit kann eine Zunahme von absolut 0,6\% (Frauen: $0,4 \%$; Männer: 0,7\%) und relativ $14 \%$ (Frauen: 9\%, Männer: 19\%) der demografischen Alterung zugeordnet werden.

Hochrechnungen der BGS98-Daten ergeben, dass vor rund 10 Jahren bei insgesamt 3,3 Mio. der 18- bis 79-Jährigen in Deutschland jemals ein Diabetes festgestellt wurde. Nach den Schätzungen, die 
Tab. 5 Lebenszeitprävalenz (Prozent, 95\%-Konfidenzintervall) des bekannten Diabetes nach Geschlecht im zeitlichen Vergleich. N(DEGS1) ungewichtet $_{\text {1 }}=7080$, N(BGS98) ungewichtet $=7099$

\begin{tabular}{|c|c|c|c|}
\hline Survey & BGS98 ${ }^{a}$ & BGS98 $^{\mathrm{b}}$ & DEGS1 ${ }^{b}$ \\
\hline \multicolumn{4}{|c|}{ Geschlecht } \\
\hline Frauen & $5,7(4,8-6,7)$ & $6,1(5,1-7,2)$ & $7,4(6,5-8,5)$ \\
\hline Männer & $4,7(4,1-5,4)$ & $5,4(4,7-6,3)$ & $7,0(6,0-8,1)$ \\
\hline Gesamt & $5,2(4,6-5,9)$ & $5,8(5,1-6,5)$ & $7,2(6,5-8,0)$ \\
\hline
\end{tabular}

auf DEGS1 basieren, wurde derzeit hingegen bei insgesamt 4,6 Mio. Personen dieser Altersgruppe jemals ein Diabetes festgestellt.

\section{Diskussion}

Den aktuellen DEGS1-Daten zufolge wurde bei 7,2\% bzw. 4,6 Mio. der 18- bis 79-jährigen Bevölkerung Deutschlands jemals ein Diabetes diagnostiziert. Die Lebenszeitprävalenz des bekannten Diabetes steigt im Altersgang deutlich an und ist bei einem niedrigen Sozialstatus höher als bei einem hohen Sozialstatus. Die Prävalenz ist bei gesetzlich Krankenversicherten, insbesondere bei AOK-Versicherten, höher als bei privat Krankenversicherten. Es gibt zudem Hinweise auf regionale Unterschiede, insbesondere hinsichtlich einer Tendenz zu einer höheren Prävalenz in den neuen im Vergleich zu den alten Bundesländern. Im Vergleich zum BGS98 ist die Prävalenz des bekannten Diabetes um 38\% angestiegen, wobei ein Anstieg um 14\% der demografischen Alterung der Bevölkerung zuzuschreiben ist.

\section{Prävalenz des bekannten Diabetes}

Die zuletzt auf Bevölkerungsebene als telefonische Befragungen bundesweit durchgeführten Gesundheitssurveys GEDA 2009 [8, 9] und GEDA 2010 [10] ergeben gepoolt eine Lebenszeitprävalenz von $8,7 \%$ bzw. eine Zahl von 5,9 Mio. Erwachsenen ab 18 Jahren mit einer jemaligen Diabetesdiagnose. Eine Begrenzung der GEDA-Daten auf die Alterspanne 18 bis 79 Jahre zeigt mit 8,2\% eine im Vergleich zu DEGS1 höhere Prävalenz. Umgekehrt resultiert aus einer in GEDA und anderen Studien beobachteten Diabetesprävalenz in der Altersgruppe ab 80 Jah- ren, die ähnlich hoch ist wie für 70 - bis 79-Jährige [20, 21, 22, 23, 24, 25, 26], bei Übertragung auf DEGS1 eine Zahl von lediglich 5,5 Mio. Erwachsenen ab 18 Jahren mit einem bekannten Diabetes. Der verbleibende Unterschied in der Größenordnung der Schätzung zwischen DEGS1 und GEDA resultiert vermutlich größtenteils aus der unterschiedlichen Stichprobenzusammensetzung aufgrund einer unterschiedlichen Stichprobenziehung (Einwohnermeldeamtsstichprobe vs. Stichprobe von über Festnetzanschluss verfügbaren Privathaushalten) und einer unterschiedlichen Erhebungsmethode (persönliches ärztliches Interview vs. Telefoninterview). Für einen direkten zeitlichen Vergleich wurden die DEGS1-Daten hier deshalb ausschließlich den zuletzt im BGS98 mit vergleichbarer Methodik erhobenen Daten gegenübergestellt.

Die bundesweit postalisch-schriftlich durchgeführten Surveys des Bertelsmann-Gesundheitsmonitors ergeben für den bekannten Diabetes in der 18bis 79-jährigen Bevölkerung für das Jahr 2008 eine Prävalenz von 8,0\% [27]. Diese liegt zwischen den ermittelten Lebenszeitprävalenzen in DEGS1 (2008-2011) mit $7,2 \%$ und GEDA (2008-2010) mit $8,2 \%$. Die Versichertenstichprobe AOK Hessen/KV Hessen weist für Versicherte aller Altersgruppen für das Jahr 2009 mit 9,8\% [5] [18- bis 79-Jährige (unter Ausschluss stationärer Pflegefälle): 10,2\%] dagegen auf eine Prävalenz hin, die zwar höher ist als die in GEDA (2008-2010) für Erwachsene ab 18 Jahren mit 8,7\% (18- bis 79-Jährige: $8,2 \%)$. Dieser erwartungsgemäße Unterschied steht jedoch in Übereinstimmung mit den vorliegenden Beobachtungen aus DEGS1 und anderen aktuellen Analysen [27, 28], denen zufolge AOK-Versicherte im Vergleich zu an- derweitig Versicherten die höchste Diabetesprävalenz aufweisen. Neben Abweichungen in der Altersstruktur, die in altersstandardisierten Analysen weitgehend berücksichtigt werden können, spielen dabei vor allem Unterschiede im Bildungsstatus und im Vorliegen von Komorbiditäten eine Rolle [29].

Weitere in $\bullet$ Tab. 6 aufgeführte Ergebnisse von Studien zum bekannten Diabetes in Deutschland sind aufgrund der Studiendurchführung vor dem DEGS1-Erhebungszeitraum nicht mit den aktuellen Ergebnissen vergleichbar. Bezüglich der relativ hohen Prävalenz des bekannten Diabetes in den Patientenstichproben haus- oder allgemeinärztlicher Praxen ist anzumerken, dass in einem Praxisklientel mehr Personen mit einem diagnostizierten Diabetes zu erwarten sind als in der Allgemeinbevölkerung. Insgesamt weisen die in $\bullet$ Tab. 6 zusammengefassten Studien ähnlich wie DEGS1 neben den bereits erwähnten Unterschieden je nach Art der Krankenversicherung - auf einen deutlichen Prävalenzanstieg mit zunehmendem Alter, insbesondere ab dem 50. Lebensjahr $[8,20,21,22,23$, $24,25,26,28,30,31,32,33,34,35,36,37]$ sowie auf soziale Unterschiede $[8,27]$ und regionale Tendenzen $[8,10,27,32]$ in der Diabetesprävalenz hin.

Differenzierte Angaben zur Prävalenz des Typ-1-Diabetes finden sich lediglich in einigen Patientenstichproben [25,37, 38]. Der Anteil an Typ-1-Diabetes an der jeweiligen Gesamtprävalenz des bekannten Diabetes liegt in diesen Stichproben zwischen rund 3\% und $6 \%$ und ist somit höher als in DEGS1 mit etwa 1\%. Neben dem unterschiedlichen Studiendesign kann bei diesem Größenunterschied die eher als konservativ einzustufende Abschätzung der Typ-1-Prävalenz in DEGS1 anhand der zur Verfügung stehenden Informationen eine Rolle spielen. Differenzierte Angaben zur Prävalenz des Schwangerschaftsdiabetes wurden in keiner der in $\bullet$ Tab. 6 zusammengefassten Studien berichtet.

\section{Zeitliche Entwicklung des bekannten Diabetes}

Die für Deutschland früheste Zeitreihe zur Prävalenz des bekannten Diabe- 
Tab. 6 Übersicht zu Studien in Deutschland mit Angaben zur Prävalenz des bekannten Diabetes bei Erwachsenen

$\begin{array}{lll}\text { Studienpopulation } & \begin{array}{l}\text { Zeitraum Diabetesdefinition } \\ \text { (Standardi- } \\ \text { sierung) }\end{array} & \end{array}$

Bundesweite Befragungs- und Untersuchungssurveys

DEGS1 Repräsentative Surveys der

18 bis 79 Jahre, $\mathrm{N}=7080 \quad$ Wohnbevölkerung Deutsch-

2008-11 Selbstangabe zu ärztlicher

Gesamt: 7,2\% (Frauen: 7,4\%, Männer: 7,0\%)

(- Tab.4, $\bullet$ Tab. 5) lands

(31.12.2010) Diabetesdiagnose oder Anti- Typ-1:0,1\%, Schwangerschaftsdiabetes/

diabetikaeinnahme

Frauen: $1,2 \%$

AOK: $9,0 \%$, EKK: 7,0\%, BKK: 5,9\%, andere

GKV: $7,9 \%$, PKV: $3,8 \%$, Sonstige: $4,7 \%$

\section{BGS98}

1997-99

Gesamt: 5,2\% (Frauen: 5,7\%, Männer: 4,7\%)

18 bis 79 Jahre, $\mathrm{N}=7099$

(31.12.1997)

(- Tab. 5) [30] ${ }^{\mathrm{a}}$

Gesundheitssurvey Ost/West 91, repräsentative Surveys der Wohnbevölkerung der neuen/alten Bundesländer

1990-92

(1991)

25 bis 69 Jahre, $\mathrm{N} \approx 7450$ [30]

Deutsche Herz-Kreislauf-Präventionsstudie, repräsentative 1990/91,

Surveys der Wohnbevölkerung der alten Bundesländer $\quad$ 1987/88,

25 bis 69 Jahre, $N \approx 5000$ (je Survey) [31] 1984-86

(1984)

Bundesweite Telefonsurveys

\begin{tabular}{|c|c|c|}
\hline $\begin{array}{l}\text { GEDA } 2010 \\
18+\text { Jahre, } \mathrm{N}=22.050 \\
{[10]}\end{array}$ & \multirow{4}{*}{$\begin{array}{l}\text { Repräsentative Surveys der } \\
\text { über Festnetzanschluss er- } \\
\text { reichbaren deutschsprachigen } \\
\text { Wohnbevölkerung Deutsch- } \\
\text { lands }\end{array}$} & $\begin{array}{l}2009 / 10 \\
(31.12 .2008)\end{array}$ \\
\hline $\begin{array}{l}\text { GEDA } 2009 \\
\text { 18+ Jahre, } N=21.262 \\
{[8,9]}\end{array}$ & & $\begin{array}{l}2008 / 09 \\
(31.12 .2007)\end{array}$ \\
\hline $\begin{array}{l}\text { GSTel03 } \\
\text { 18+ Jahre, N=8318 }[8,9]\end{array}$ & & $\begin{array}{l}2002 / 03 \\
(31.12 .2001)\end{array}$ \\
\hline $\begin{array}{l}\text { GSTel03, GSTel04 (ge- } \\
\text { poolt) }\end{array}$ & & $\begin{array}{l}2002-04 \\
(31.12 .2001)\end{array}$ \\
\hline
\end{tabular}

18+ Jahre, $\mathrm{N}=15.354$

[28]

Bundesweite postalische Surveys

Bertelsmann-Gesundheitsmonitor, repräsentative Surveys 2004-08

der deutschsprachigen Wohnbevölkerung Deutschlands

18 bis 79 Jahre, $N=15.089(N \approx 1500$ je Survey) [27]
Selbstangabe zur Diabetes- Gesamt 6,9\% (Frauen: 5,8\%, Männer: 8,0\%) diagnose ( $\geq 1$ Arztbesuch pro $\quad 2008: 8,0 \%, 2007: 7,2 \%, 2006: 6,5 \%, 2005$ : Quartal oder regelmäßige $\quad 6,4 \%, 2004: 6,2 \%$ Medikamenteneinnahme) $\quad$ AOK: $11,4 \%$, Barmer: $8,5 \%$, DAK: $6,5 \%$, BKK: 5,9\%, TKK: $5,8 \%$, PKV: 4,6\%, IKK: 3,9\%, Sonstige: $5,2 \%$

\section{Registerdaten}

Diabetesregister der ehemaligen DDR

Alle Altersgruppen, gesamte Bevölkerung [20,39]

1960-89 Ärztliche Diabetesdiagnose

1989: $4,1 \%$

1987: $4,0 \%$

1960: 0,6\%

Diabetesregister der ehemaligen DDR

1960-89

1988: 3,8\% (Frauen: 4,5\%, Männer: 3,0\%) 1970: $2,5 \%$

(47]

Regionale Studien

Landgesundheitsstudie, Stichproben aus zufällig ausge-

wählten nordostdeutschen Landgemeinden

2004/08, Selbstangabe zu Diabetes-

1994, 1973 diagnose

(2004/08)

(1973) [48]

DIAB-CORE Consortium mit dem Survey BGS98 (1997-99)

und den regionalen Studien CARLA (2002-06), DO-GS

(2003/04), HNR (2000-03), KORA S4 (1999-2001), SHIP

(1997-2001)

45 bis 74 Jahre, $\mathrm{N}=15.071$ [32]

KORA F4 (Follow-up), Kohortenstudie im Raum Augsburg 35 bis 59 Jahre, $\mathrm{N}=1653$ [33]

KORA S4, Survey im Raum Augsburg

55 bis 74 Jahre, $\mathrm{N}=1353$ [34]

1997-2006

(31.12.2007)

Selbstangabe zu Diabetes-

diagnose oder Antidiabetikaeinnahme und Alter bei Diabetesdiagnose $>30$ Jahre

2004/08: Frauen: 12,4\%, Männer: 12,8\%

1994: Frauen: 10,9\%, Männer: 6,8\%

1973: Frauen: 3,5\%, Männer: 3,1\%

Typ-2: $8,6 \%$

BGS98: $8,2 \%$, CARLA: $12,0 \%$, DO-GS: $9,3 \%$,

HNR: $7,2 \%$, KORA: $5,8 \%$, SHIP: $10,9 \%$

2006-08 Selbstangabe zu ärztlicher

(31.12.2007) Diabetesdiagnose oder

1999-2001 Antidiabetikaeinnahme

(31.12.2000) (verifiziert)
Gesamt: 2,2\% (Frauen: 2,3\%, Männer: 2,2\%)

Gesamt: 8,7\% (Frauen: 8,0\%, Männer: 9,3\%) 
Tab. 6 Übersicht zu Studien in Deutschland mit Angaben zur Prävalenz des bekannten Diabetes bei Erwachsenen (Fortsetzung)

\begin{tabular}{|c|c|c|c|}
\hline Studienpopulation & $\begin{array}{l}\text { Zeitraum } \\
\text { (Standardi- } \\
\text { sierung) }\end{array}$ & Diabetesdefinition & Diabetesprävalenz \\
\hline $\begin{array}{l}\text { MONICA/KORA, Surveys im Raum Augsburg } \\
25 \text { bis } 64 \text { Jahre (19984/85), } 25 \text { bis } 74 \text { Jahre (weitere } \\
\text { Surveys), } N \approx 2000 \text { (je Survey) [35] }\end{array}$ & $\begin{array}{l}\text { 1999-2001, } \\
1994 / 95 \\
1989 / 90 \\
1984 / 85 \\
(31.12 .2000)\end{array}$ & $\begin{array}{l}\text { Selbstangabe zu ärztlicher } \\
\text { Diabetesdiagnose oder Anti- } \\
\text { diabetikaeinnahme }\end{array}$ & $\begin{array}{l}25 \text { bis } 64 \text { Jahre: } \\
\text { 1999-2001: Frauen: 2,7\%, Männer: 2,5\% } \\
\text { 1994/95: Frauen: 2,5\%, Männer: 3,0\% } \\
\text { 1989/90: Frauen: 2,3\%, Männer: 3,6\% } \\
\text { 1984/85: Frauen: 2,0\%, Männer: 2,7\% } \\
25 \text { bis 74 Jahre: } \\
\text { 1999-2001: Frauen: 3,5\%, Männer: 3,7\% } \\
\text { 1994/95: Frauen: 3,7\%, Männer: 4,1\% } \\
\text { 1989/90: Frauen: 3,6\%, Männer: 4,6\% }\end{array}$ \\
\hline $\begin{array}{l}\text { EPIC-Potsdam, Kohorten-Basisuntersuchung im Raum } \\
\text { Potsdam } \\
35 \text { bis } 59 \text { Jahre, } \mathrm{N} \approx 27.500 \text { [11] }\end{array}$ & $\begin{array}{l}1994-98 \\
(2007)\end{array}$ & $\begin{array}{l}\text { Selbstangabe zu ärztlicher } \\
\text { Diabetesdiagnose oder Dia- } \\
\text { betestherapie }\end{array}$ & Frauen: $2,6 \%$, Männer: 3,7\% \\
\hline $\begin{array}{l}\text { Stichprobe aus zufällig ausgewählten Städten und länd- } \\
\text { lichen Bezirken in } 5 \text { Bundesländern } \\
18 \text { bis } 70 \text { Jahre, } N=2150 \text { [36] }\end{array}$ & 1993-96 & $\begin{array}{l}\text { Selbstangabe zu Diabetes- } \\
\text { diagnose oder Diabetes- } \\
\text { therapie }\end{array}$ & Gesamt: 6,3\% \\
\hline $\begin{array}{l}\text { Münchener Diabetes-Früherfassung } \\
\text { Alle Altersgruppen, } \mathrm{N} \approx 790.000 \text { [49] }\end{array}$ & $1967 / 68$ & $\begin{array}{l}\text { Selbstangabe zu Diabetes- } \\
\text { diagnose }\end{array}$ & Gesamt: 2,0\% \\
\hline Krankenversichertendaten & & & \\
\hline $\begin{array}{l}\text { Versichertenstichprobe AOK Hessen/KV Hessen } \\
\text { Alle Altersgruppen, } N \approx 300.000 \text { pro Jahr [5] }\end{array}$ & $\begin{array}{l}2000-09 \\
(31.12 .2009)\end{array}$ & \multirow{3}{*}{$\begin{array}{l}\text { Ärztliche Diabetesdiagnose } \\
\text { (in } \geq 3 \text { von } 4 \text { Quartalen) oder } \\
\text { Antidiabetikaverordnung ( } \geq 2 \\
\text { pro Jahr oder } 1 \text { pro Jahr plus } \\
\text { Diabetesdiagnose oder plus } \\
\text { Glukose- oder HbA1c-Mes- } \\
\text { sung im selben Quartal) }\end{array}$} & $\begin{array}{l}2009: 9,8 \% \\
2000: 7,5 \%\end{array}$ \\
\hline $\begin{array}{l}\text { Versichertenstichprobe AOK Hessen/KV Hessen } \\
\text { Alle Altersgruppen, } \mathrm{N} \approx 300.000 \text { pro Jahr [50] }\end{array}$ & $\begin{array}{l}\text { 2000-07 } \\
\text { (31.12. des } \\
\text { Vorjahres) }\end{array}$ & & $\begin{array}{l}2007: 8,9 \% \\
2000: 6,5 \%\end{array}$ \\
\hline $\begin{array}{l}\text { Versichertenstichprobe AOK Hessen/KV Hessen } \\
\text { Alle Altersgruppen, } \mathrm{N} \approx 300.000 \text { pro Jahr }[21,22]\end{array}$ & $\begin{array}{l}\text { 1998-2004 } \\
\text { (31.12. des } \\
\text { Vorjahres) }\end{array}$ & & $\begin{array}{l}\text { 2004: 7,9\% (Frauen: 8,1\%, Männer 7,6\%) } \\
\text { 2001: 6,9\% (Frauen: 7,4\%, Männer: 6,5\%) } \\
\text { 1998:5,9\% }\end{array}$ \\
\hline $\begin{array}{l}\text { Versichertenstichprobe von } 6 \text { gesetzlichen Kranken- } \\
\text { versicherungen } \\
\text { Alle Altersgruppen, } N=14,7 \text { Mio. [23] }\end{array}$ & 1999 & $\begin{array}{l}\text { Diabetesdiagnose oder Anti- } \\
\text { diabetikaverordnung }\end{array}$ & Gesamt: 6,5\% \\
\hline $\begin{array}{l}\text { Versichertenstichprobe AOK-Dortmund } \\
\text { Alle Altersgruppen, } \mathrm{N}=6478 \text { [24] }\end{array}$ & $\begin{array}{l}1988 \\
(1988)\end{array}$ & $\begin{array}{l}\text { Ärztliche Diabetesdiagnose } \\
\text { (in } \geq 2 \text { von } 4 \text { Quartalen) oder } \\
\text { Antidiabetikaverordnung } \\
\text { ( } \geq 4 \text { pro Jahr) oder Blut- } \\
\text { zuckermessung (in } \geq 3 \text { von } \\
\text { 4 Quartalen) }\end{array}$ & Gesamt: 4,8\% (Frauen: 5,5\%, Männer: 4,1\%) \\
\hline \multicolumn{4}{|l|}{ Patientendaten } \\
\hline $\begin{array}{l}\text { GEMCAS, nationale Patientenstichprobe hausärztlicher } \\
\text { Praxen } \\
18+\text { Jahre, } N=35.869 \text { ( } N=1511 \text { Praxen) [25] }\end{array}$ & $\begin{array}{l}2005 \\
(2003)\end{array}$ & Ärztliche Diabetesdiagnose & $\begin{array}{l}\text { Gesamt: } 11,8 \% \\
\text { Typ-1:0,7\%, Typ-2: } 11,1 \%\end{array}$ \\
\hline $\begin{array}{l}\text { DETECT-Studie, nationale Stichprobe von Allgemeinarzt- } \\
\text { praxen } \\
18+\text { Jahre, } \mathrm{N}=55.518 \text { ( } \mathrm{N}=3188 \text { Praxen) [37] }\end{array}$ & 2003 & $\begin{array}{l}\text { Ärztliche Angabe zur Diabe- } \\
\text { tesdiagnose am Studientag } \\
\text { oder Antidiabetikaeinnahme }\end{array}$ & $\begin{array}{l}\text { Gesamt: } 15,2 \% \\
\text { Typ-1: 0,5\%, Typ-2: } 14,7 \%\end{array}$ \\
\hline $\begin{array}{l}\text { ESTHER, Patientenstichprobe aus Gesundheits-Check-ups } \\
\text { hausärztlicher Praxen im Saarland } \\
50 \text { bis } 74 \text { Jahre, N=9953 [51] }\end{array}$ & $2000-02$ & $\begin{array}{l}\text { Ärztliche Diabetesdiagnose } \\
\text { oder Antidiabetikaeinnahme }\end{array}$ & Gesamt: $13,8 \%$ \\
\hline $\begin{array}{l}\text { HYDRA-Studie, nationale Patientenstichprobe allgemein- } \\
\text { ärztlicher Praxen/hausärztlich tätiger Internisten } \\
\text { 16+ Jahre, } N=43.549 \text { ( } N=1912 \text { Praxen) [26] }\end{array}$ & 2001 & $\begin{array}{l}\text { Ärztliche Angabe zur Diabe- } \\
\text { tesdiagnose am Studientag }\end{array}$ & $\begin{array}{l}\text { Gesamt:15,6\% (Frauen: 13,7\%, Männer: } \\
\text { 18,5\%) }\end{array}$ \\
\hline $\begin{array}{l}\text { SESAM 2, Patientenstichprobe allgemeinärztlicher Praxen } \\
\text { in Sachsen } \\
2 \text { bis } 102 \text { Jahre, } N=8877 \text { ( } N=270 \text { Praxen) [38] }\end{array}$ & $\begin{array}{l}1999 / 2000 \\
(2000)\end{array}$ & Ärztliche Diabetesdiagnose & $\begin{array}{l}\text { Gesamt: 9,2\% } \\
\text { Typ-1: 0,3\%, Typ-2: 6,2\%, andere Typen: } \\
\text { 1,8\%, nicht weiter spezifizierte Typen: 1,0\% }\end{array}$ \\
\hline
\end{tabular}


tes beruht auf Daten des zwischen 1960 und 1989 existierenden Diabetesregisters der ehemaligen DDR. Für diesen Zeitraum wurde ein kontinuierlicher Prävalenzanstieg von $0,6 \%$ auf $4,1 \%$ beobachtet, der größtenteils auf den Prävalenzanstieg in den Altersgruppen ab 50 Jahren zurückzuführen war [20,39]. Daten zum Prävalenztrend in den alten Bundesländern existieren für diesen Zeitraum nicht.

Für den nachfolgenden Zeitraum bis etwa 2000 lässt sich auf Datengrundlage bevölkerungsbezogener Studien kein Hinweis auf einen weiteren Prävalenzanstieg finden. So wurde weder in den Surveys der Deutschen Herz-KreislaufPräventionsstudie in den alten Bundesländern für 25- bis 69-Jährige zwischen 1984-1986 und 1990/1991 [31] noch in den KORA/MONICA-Surveys im Raum Augsburg für 25- bis 64-Jährige zwischen 1984/1985 und 1999-2001 bzw. für 25- bis 74-Jährige zwischen 1989/90 und 19992001 ein Trend in der Diabetesprävalenz beobachtet [35]. Auch der Vergleich von Daten der bundesweiten Untersuchungssurveys zeigt für die 25- bis 69-jährige Bevölkerung keinen Prävalenzanstieg zwischen 1990-1992 und 1997-1999 [30] weder für die neuen noch für die alten Bundesländer [40]. Eine Erweiterung dieses zeitlichen Vergleichs um Daten der telefonischen Gesundheitssurveys von 2002-2005 lässt ebenfalls keinen Trend erkennen [40].

Der in der vorliegenden Analyse dargestellte Vergleich von Daten aus DEGS1 mit denen aus BGS98 weist in Übereinstimmung mit anderen aktuellen Untersuchungen auf einen Prävalenzanstieg des bekannten Diabetes innerhalb der letzten Dekade hin. So kann für die 18bis 79-jährige Bevölkerung anhand der Untersuchungssurveys von 1997-1999 bis 2008-2011 ein Anstieg um 38\% und anhand der Surveys des BertelsmannGesundheitsmonitors von 2004 bis 2008 ein Anstieg um 29\% [27] ermittelt werden. Weiterhin kann für Erwachsene ab 18 Jahren aufgrund der telefonischen Surveys von 2002/2003 bis 20082010 ein Anstieg um 43\% und aufgrund der Versichertenstichprobe AOK Hessen/KV Hessen von 2000 bis 2009 ein Anstieg um 49\% [5] beobachtet werden. Dabei zeigt sich bei Männern ein stärke- rer Anstieg als bei Frauen: In den Untersuchungssurveys beträgt dieser $49 \%$ vs. $30 \%$, in den telefonischen Surveys 54\% vs. 34\% und in der Versichertenstichprobe $57 \%$ vs. $40 \%$. Zudem ergibt die Datenanalyse der Untersuchungssurveys, dass übereinstimmend mit Ergebnissen der Versichertenstichprobe AOK Hessen/KV Hessen [5] etwa ein Drittel des beobachteten Anstiegs auf die demografische Alterung innerhalb des Beobachtungszeitraums zurückzuführen ist.

Schätzungen der Prävalenz des bekannten Typ-2-Diabetes für das Jahr 2030 auf Basis der Diabetesprävalenz bevölkerungsbezogener regionaler Studien und des BGS98 (DIAB-CORE Verbund), der Diabetesinzidenz und -mortalität in der Region Augsburg (KORA S4/F4) und der Bevölkerungsvorausberechnung des Statistischen Bundesamtes ergeben allein für die 55- bis 74-Jährigen gegenüber heute einen Anstieg um etwa 1,5 Mio. Personen (+64\%) [41]. Für Männer wird mit fast einer Millionen Personen ein deutlicherer Zuwachs (+79\%) projiziert als für Frauen mit etwas mehr als 0,5 Mio. Personen $(+47 \%)$.

\section{Relevante Faktoren zur Interpretation der Prävalenz des bekannten Diabetes}

Die Größenordnung des bekannten Diabetes ist abhängig vom Verhältnis diagnostizierter zu nicht diagnostizierten Fällen. Vereinzelt durchgeführte Analysen zur Einschätzung dieses Verhältnisses kommen aufgrund unterschiedlicher Studienpopulationen und Erhebungszeiträume und insbesondere aufgrund unterschiedlicher Diagnosekriterien zu verschiedenen Resultaten. So weisen Ergebnisse des oralen Glukosetoleranztests der KORA-Studie im Raum Augsburg auf eine Prävalenz des nicht diagnostizierten Diabetes (definiert als Nüchtern-Glukose $\geq 7,0 \mathrm{mmol} / \mathrm{l}$ oder Glukose nach oralem Glukosetoleranztest $\geq 11,1 \mathrm{mmol} / \mathrm{l}$ ) hin, die ebenso hoch ist wie die des diagnostizierten Diabetes [33, 34]. Erste Auswertungen aus DEGS1, in der kein oraler Glukosetoleranztest durchgeführt wurde, jedoch Laborwerte für Nüchtern- ( $48 \%$ der Stichprobe) bzw. Gelegenheitsglukose und für HbAlc vorlie- gen, deuten dagegen auf eine Prävalenz des nicht diagnostizierten Diabetes (definiert als $\mathrm{HbAlc} \geq 6,5 \%$ oder Nüchternglukose $\geq 7,0 \mathrm{mmol} / \mathrm{l}$ oder Gelegenheitsglukose $\geq 11,1 \mathrm{mmol} / \mathrm{l}$ ) hin [42], die mehr als dreifach geringer ist als die des bekannten Diabetes. In früheren Studien, in denen kein oraler Glukosetoleranztest durchgeführt wurde, lag die Prävalenz des nicht diagnostizierten Diabetes basierend auf verschiedenen Kriterien im Vergleich zur Prävalenz des diagnostizierten Diabetes ebenfalls deutlich niedriger [25, 30,36].

Das Verhältnis des diagnostizierten zum nicht diagnostizierten Diabetes kann sich im Verlauf der Zeit verschieben. So können verbesserte Früherkennungsmaßnahmen, eine erhöhte Aufmerksamkeit seitens der Ärzte (beispielsweise durch Einführung der Disease-Management-Programme) und eine verbesserte Kenntnis der Symptome seitens der Patienten zu einer früheren Entdeckung von Personen mit Diabetes führen und folglich den Anteil diagnostizierter Fälle erhöhen, während der Anteil nicht diagnostizierter Fälle gleichermaßen sinkt. Daher sind Daten zum Trend des nicht diagnostizierten Diabetes, die über die Zeit mit vergleichbarer Methodik erhoben wurden, für die Interpretation des beobachteten Trends des bekannten Diabetes unerlässlich. Solche Daten existieren für Deutschland jedoch bisher nicht [11]. Vorgesehene vergleichende Analysen von DEGS1 und BGS98 werden hier erstmals einen Anhaltspunkt liefern können.

Die Prävalenz des bekannten Diabetes - und auch des nicht diagnostizierten Diabetes - ist weiterhin von der Inzidenz- und Mortalitätsrate abhängig. Die Inzidenzrate steht in engem Zusammenhang mit Verhaltensweisen (wie Ernährung, körperliche Aktivität und damit assoziiertes Körpergewicht) und Lebensverhältnissen (wie persönliche oder regionale wirtschaftliche Faktoren, Luftverschmutzung), die bei der Diabetesentwicklung eine Rolle spielen [2]. So ist durch die beobachtete Zunahme der Adipositas [43], dem Hauptrisikofaktor des Diabetes, in der Folge ein Anstieg der Diabetesinzidenz wahrscheinlich. Die Mortalitätsrate bei Personen mit Diabetes ist zusätzlich zur allgemeinen Überlebenszeit mit der Behandlungsintensi- 
tät bzw. dem Behandlungserfolg assoziiert. So wäre bei einer höheren allgemeinen Lebenserwartung und verbesserten Behandlungsmöglichkeiten ebenfalls mit einer höheren Prävalenz zu rechnen. Bevölkerungsbezogene Daten zur Inzidenz und Mortalität liegen bisher außer aus dem Diabetesregister der ehemaligen DDR nur aus dem Raum Augsburg vor $[44,45]$. Auch zur Abschätzung der bundesweiten Diabetesinzidenz und -mortalität und deren zeitlicher Entwicklung werden die Monitoring-Daten des RKI einen Beitrag leisten können.

\section{Stärken und Limitationen}

Die Stärken der vorliegenden Untersuchung liegen in der Bevölkerungsrepräsentativität der Aussagen für die deutsche Wohnbevölkerung im Alter von 18 bis 79 Jahren und in der Vergleichbarkeit der Prävalenzen zu 2 Zeitpunkten im Abstand von mehr als 10 Jahren aufgrund gleicher Stichprobenziehung und gleicher Definition des bekannten Diabetes. Prävalenzschätzungen und Hochrechnungen für die Bevölkerung werden durch Gewichtung der Ergebnisse mit komplexen Gewichtungsfaktoren ermöglicht, die das komplexe Studiendesign, Non-Response und Wiederteilnahmebereitschaft von BGS98-Teilnehmenden berücksichtigen $[15,16]$. Die Limitationen der vorliegenden Arbeit bestehen darin, dass die Bevölkerung ab 80 Jahren sowie bestimmte Personengruppen (insbesondere Pflegeheimbewohner, Schwerkranke) nicht repräsentativ in den Untersuchungssurvey einbezogen werden können. Zudem ist keine umfassende Beurteilung des Krankheitsgeschehens möglich, da sich die vorliegenden Schätzungen auf den bereits diagnostizierten Diabetes beschränken. Weiterhin beruht die Definition des bekannten Diabetes lediglich auf Selbstangaben der Teilnehmenden, die jedoch als relativ valide Quelle zur Erfassung eines diagnostizierten Diabetes angesehen werden können [46].

\section{Fazit und Ausblick}

Die aktuellen DEGS1-Ergebnisse weisen im Kontext mit anderen bundeswei- ten Surveys auf eine Zahl von mindestens 4,6 Mio. 18- bis 79-jährigen Erwachsenen mit einer jemaligen Diabetesdiagnose in Deutschland hin. Dabei sind insbesondere Personen ab dem 50. Lebensjahr und Personen mit einem niedrigen Sozialstatus betroffen. Innerhalb der letzten Dekade ist die Zahl der Erwachsenen mit einem bekannten Diabetes um 38\% angestiegen. Davon ist etwa ein Drittel auf die demografische Alterung der Bevölkerung zurückzuführen. Geplante Analysen zur zeitlichen Entwicklung des nicht diagnostizierten Diabetes sowie zur Inzidenz- und Mortalitätsrate werden zur Interpretation dieser Ergebnisse beitragen.

\section{Korrespondenzadresse}

\section{Dr. C. Heidemann}

Abteilung für Epidemiologie und

Gesundheitsmonitoring, Robert Koch-Institut

General-Pape-Str. 62-66,

12101 Berlin

HeidemannC@rki.de

Danksagungen. Wir danken R. Paprott für die Unterstützung bei der Literaturrecherche.

Finanzierung der Studie. Die Studie wurde finanziert mit Mitteln des Robert Koch-Instituts und des Bundesministeriums für Gesundheit. Diese Arbeit wurde unterstützt durch Mittel vom „Kompetenznetz Diabetes mellitus" des Bundesministeriums für Bildung und Forschung, FKZ 01GI1110F.

Interessenkonflikt. Die korrespondierende Autorin weist für sich und ihre Koautoren auf folgende Beziehungen hin: Dr. W. Rathmann hat im Rahmen von Vorträgen und Beratertätigkeiten Honorare von den folgenden Firmen erhalten: BMS, Eli Lilly, NovoNordisk, IMS HEALTH und Sanofi-Aventis. Dr. C. Heidemann, Dr. Y. Du, Dr. I. Schubert und Dr. C. Scheidt-Nave geben an, dass kein Interessenkonflikt besteht.

\section{Literatur}

1. American Diabetes Association (2012) Diagnosis and classification of diabetes mellitus. Diabetes Care 35(Suppl 1):64-71

2. Chen L, Magliano DJ, Zimmet PZ (2012) The worldwide epidemiology of type 2 diabetes mellitus - present and future perspectives. Nat Rev Endocrinol 8:228-236

3. Giani G, Janka HU, Hauner H et al (2004) Epidemiologie und Verlauf des Diabetes mellitus in Deutschland. In: Scherbaum WA, Kiess W (Hrsg) Evidenzbasierte Leitlinie DDG

4. Schunk M, Reitmeir P, Schipf S et al (2012) Healthrelated quality of life in subjects with and without Type 2 diabetes: pooled analysis of five population-based surveys in Germany. Diabet Med 29:646-653
5. Koster I, Schubert I, Huppertz E (2012) Follow up of the CoDiM-Study: cost of diabetes mellitus 2000-2009. Dtsch Med Wochenschr 137:10131016

6. Danaei G, Finucane MM, Lu Y et al (2011) National, regional, and global trends in fasting plasma glucose and diabetes prevalence since 1980: systematic analysis of health examination surveys and epidemiological studies with 370 country-years and 2.7 million participants. Lancet 378:31-40

7. International Diabetes Federation (2011) IDF Diabetes Atlas, 5th edt. International Diabetes Federation, Brussels

8. Heidemann C, Du Y, Scheidt-Nave C (2011) Diabetes mellitus in Deutschland. In: GBE kompakt. Robert-Koch-Institut, Berlin. http://www.rki.de/gbekompakt

9. Robert Koch-Institut (RKI) (2010) Daten und Fakten: Ergebnisse der Studie "Gesundheit in Deutschland aktuell 2009". RKI, Berlin

10. Robert Koch-Institut (RKI) (2012) Daten und Fakten: Ergebnisse der Studie "Gesundheit in Deutschland aktuell 2010". RKI, Berlin

11. Schulze MB, Rathmann W, Giani G, Joost HG (2010) Diabetesprävalenz - Verlässliche Daten stehen noch aus. Dtsch Arztebl 107:A1694-A1696

12. Kurth BM, Lange C, Kamtsiuris P, Hölling H (2009) Gesundheitsmonitoring am Robert Koch-Institut. Bundesgesundheitsbl Gesundheitsforsch Gesundheitsschutz 52:557-570

13. Kurth BM (2012) Das RKI-Gesundheitsmonitoring - was es enthält und wie es genutzt werden kann. Public Health Forum 20(76):4.e1-4.e3

14. Gößwald $A$, Lange $M$, Kamtsiuris $P$, Kurth BM (2012) DEGS: Studie zur Gesundheit Erwachsener in Deutschland. Bundesweite Quer- und Längsschnittstudie im Rahmen des Gesundheitsmonitorings des Robert Koch-Instituts. Bundesgesundheitsbl Gesundheitsforsch Gesundheitsschutz 55:775-780

15. Scheidt-Nave C, Kamtsiuris $P$, Gößwald A et al (2012) German health interview and examination survey for adults (DEGS) - design, objectives and implementation of the first data collection wave. BMC Public Health 12:730

16. Kamtsiuris $P$, Lange $M$, Hoffmann R et al (2013) Die erste Welle der Studie zur Gesundheit Erwachsener in Deutschland (DEGS1). Stichprobendesign, Response, Gewichtung und Repräsentativität. Bundesgesundheitsbl Gesundheitsforsch Gesundheitsschutz 56:620-630

17. Robert Koch-Institut (2009) DEGS: Studie zur Gesundheit Erwachsener in Deutschland. Projektbeschreibung. Beiträge zur Gesundheitsberichterstattung des Bundes. RKI, Berlin

18. Gößwald A, Lange M, Dölle R, Hölling H (2013) Die erste Welle der Studie zur Gesundheit Erwachsener in Deutschland (DEGS1). Gewinnung von Studienteilnehmenden, Durchführung der Feldarbeit und Qualitätsmanagement. Bundesgesundheitsbl Gesundheitsforsch Gesundheitsschutz 56:611619

19. Lampert T, Kroll L, Müters S, Stolzenberg H (2013) Messung des sozioökonomischen Status in der Studie zur Gesundheit Erwachsener in Deutschland (DEGS1). Bundesgesundheitsbl Gesundheitsforsch Gesundheitsschutz 56:631-636

20. Michaelis D, Jutzi E (1991) Epidemiologie des Diabetes mellitus in der Bevölkerung der ehemaligen DDR: Alters- und geschlechtsspezifische Inzidenzund Prävalenztrends im Zeitraum 1960-1987. Z Klin Med 46:59-64 
21. Hauner H, Köster I, Schubert I (2007) Prevalence of diabetes mellitus and quality of care in hesse, Germany, 1998-2004. Dtsch Arztebl 104:A2799A2805

22. Hauner H, Koster I, Ferber L von (2003) Prevalence of diabetes mellitus in Germany 1998-2001. Secondary data analysis of a health insurance sample of the AOK in Hesse/KV in Hesse. Dtsch Med Wochenschr 128:2632-2637

23. Stock SA, Redaelli M, Wendland G et al (2006) Diabetes - prevalence and cost of illness in Germany: a study evaluating data from the statutory health insurance in Germany. Diabet Med 23:299-305

24. Hauner H, Ferber L von, Koster I (1992) Estimation of the incidence of diabetes in the Federal Republic of Germany based on insurance data. A secondary data analysis of a representative random sample of locally insured persons in the city of Dortmund. Dtsch Med Wochenschr 117:645650

25. Hauner H, Hanisch J, Bramlage P et al (2008) Prevalence of undiagnosed Type-2-diabetes mellitus and impaired fasting glucose in German primary care: data from the German Metabolic and Cardiovascular Risk Project (GEMCAS). Exp Clin Endocrinol Diabetes 116:18-25

26. Lehnert H, Wittchen HU, Pittrow D et al (2005) Prevalence and pharmacotherapy of diabetes mellitus in primary care. Dtsch Med Wochenschr 130:323-328

27. Hoffmann F, Icks A (2011) Diabetes prevalence based on health insurance claims: large differences between companies. Diabet Med 28:919-923

28. Hoffmann F, Icks A (2012) Diabetes „epidemic" in Germany? A critical look at health insurance data sources. Exp Clin Endocrinol Diabetes 120:410415

29. Hoffmann F, Icks A (2012) Structural differences between health insurance funds and their impact on health services research: results from the Bertelsmann Health-Care Monitor. Gesundheitswesen 74:291-297

30. Thefeld W (1999) Prevalence of diabetes mellitus in the adult German population. Gesundheitswesen 61(Spec No):85-89

31. Helmert U, Janka HU, Strube H (1994) Epidemiologic findings for diabetes mellitus prevalence in the Federal Republic of Germany between 1984 and 1991. Diab Stoffw 3:271-277

32. Schipf S, Werner A, Tamayo T et al (2012) Regional differences in the prevalence of known type 2 diabetes mellitus in $45-74$ years old individuals: results from six population-based studies in Germany (DIAB-CORE Consortium). Diabet Med 29:e88e95

33. Meisinger C, Strassburger K, Heier M et al (2010) Prevalence of undiagnosed diabetes and impaired glucose regulation in 35-59-year-old individuals in Southern Germany: the KORA F4 Study. Diabet Med 27:360-362

34. Rathmann W, Haastert B, Icks A et al (2003) High prevalence of undiagnosed diabetes mellitus in Southern Germany: target populations for efficient screening. The KORA survey 2000. Diabetologia 46:182-189

35. Meisinger C, Heier M, Doering A et al (2004) Prevalence of known diabetes and antidiabetic therapy between 1984/1985 and 1999/2001 in southern Germany. Diabetes Care 27:2985-2987
36. Palitzsch K-D, Nusser J, Arndt H et al (1999) The prevalence of diabetes mellitus is significantly underestimated in Germany, according to a nationwide epidemiological study based on $\mathrm{HbA} 1 \mathrm{c}$ analysis Diab Stoffw 8:189-200

37. Pittrow D, Stalla GK, Zeiher AM et al (2006) Prevalence, drug treatment and me677olic control of diabetes mellitus in primary care. Med Klin (Munich) 101:635-644

38. Frese T, Sandholzer H, Voigt S, Voigt R (2008) Epidemiology of diabetes mellitus in German general practitioners' consultation - results of the SESAM 2-study. Exp Clin Endocrinol Diabetes 116:326328

39. Michaelis D, Jutzi E, Vogt L (1993) Epidemiology of insulin-treated diabetes mellitus in the East-German population: differences in long-term trends between incidence and prevalence rates. Diabet Metab 19:110-115

40. Heidemann C, Kroll L, Icks A et al (2009) Prevalence of known diabetes in German adults aged 2569 years: results from national health surveys over 15 years. Diabet Med 26:655-658

41. Brinks R, Tamayo T, Kowall B, Rathmann W (2012) Prevalence of type 2 diabetes in Germany in 2040: estimates from an epidemiological model. Eur J Epidemiol 27:791-797

42. Heidemann C, Du Y, Scheidt-Nave C (2012) Diabetes mellitus: Wie hoch ist die Zahl der Erwachsenen mit Diabetes in Deutschland? Bundesgesundheitsbl Gesundheitsforsch Gesundheitsschutz 55:983-984

43. Mensink GB, Schienkiewitz A, Haftenberger M et al (2013) Übergewicht bei Erwachsenen in Deutschland aktuell und im Zeitverlauf. Ergebnisse der Studie zur Gesundheit Erwachsener in Deutschland (DEGS1). Bundesgesundheitsbl Gesundheitsforsch Gesundheitsschutz 56:786-794

44. Rathmann W, Strassburger K, Heier M et al (2009) Incidence of Type 2 diabetes in the elderly German population and the effect of clinical and lifestyle risk factors: KORA S4/F4 cohort study. Diabet Med 26:1212-1219

45. Kowall B, Rathmann W, Heier M et al (2011) Categories of glucose tolerance and continuous glycemic measures and mortality. Eur J Epidemiol 26:637-645

46. Midthjell K, Holmen J, Bjorndal A, Lund-Larsen G (1992) Is questionnaire information valid in the study of a chronic disease such as diabetes? The Nord-Trondelag diabetes study. J Epidemiol Community Health 46:537-542

47. Ratzmann KP (1991) An analysis og age-specific and sex-specific diabetes prevalence and its mode of treatment: The Berlin Study. Akt Endokr Stoffw 12:220-223

48. Elkeles T, Beck D, Roding D et al (2012) Health and lifestyle in rural northeast Germany: the findings of a Rural Health Study from 1973, 1994, and 2008. Dtsch Arztebl Int 109:285-292

49. Mehnert $H$, Sewering $H$, Reichstein $W$, Vogt $H$ (1968) Early detection of diabetics in Munich 1967-1968. Dtsch Med Wochenschr 93:20442050

50. Köster I, Huppertz E, Hauner H, Schubert I (2011) Direct costs of diabetes mellitus in Germany - CoDiM 2000-2007. Exp Clin Endocrinol Diabetes 119:377-385

51. Maatouk I, Wild B, Wesche D et al (2012) Temporal predictors of health-related quality of life in elderly people with diabetes: results of a German cohort study. PloS one 7:e31088 